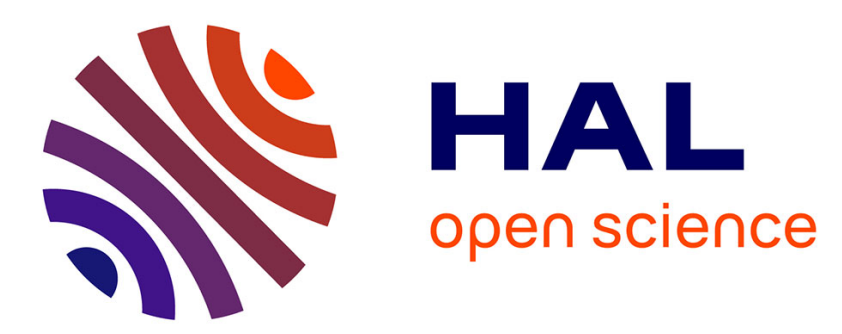

\title{
Markovian approach using several Gibbs energy for remote sensing images segmentation
}

\author{
Youssef Chahir, Sadia Alkama, Daoud Berkani
}

\section{To cite this version:}

Youssef Chahir, Sadia Alkama, Daoud Berkani. Markovian approach using several Gibbs energy for remote sensing images segmentation. Analog Integrated Circuits and Signal Processing, 2011, 69 (1), pp.39 - 47. 10.1007/s10470-011-9631-8 . hal-01882790

\section{HAL Id: hal-01882790 \\ https://hal.science/hal-01882790}

Submitted on 27 Sep 2018

HAL is a multi-disciplinary open access archive for the deposit and dissemination of scientific research documents, whether they are published or not. The documents may come from teaching and research institutions in France or abroad, or from public or private research centers.
L'archive ouverte pluridisciplinaire HAL, est destinée au dépôt et à la diffusion de documents scientifiques de niveau recherche, publiés ou non, émanant des établissements d'enseignement et de recherche français ou étrangers, des laboratoires publics ou privés. 


\title{
Markovian approach using several Gibbs energy for remote sensing images segmentation
}

\author{
Sadia Alkama $\cdot$ Youssef Chahir · Daoud Berkani
}

\begin{abstract}
The high resolution multispectral imagery needs to be segmented into regions that can be easily interpreted and which correspond roughly to the "ground truth". In this paper, we segment multispectral images MSG2, provided by meteorological satellite "Meteosat Second Generation 2", by using an approach based on support vector Markov model witch takes into account both the spectral and the spatial information. A multi-variable Gaussian distribution is used in image processing and the Gibbs energy is used to describe the process of labeling. There are several forms of Gibbs energy. We test the best known of them and evaluate the different results using the Borsotti function which is known to be more appropriate with our visual perception.
\end{abstract}

Keywords Markov fields · Vector segmentation · Evaluation function - Remote sensing images . Gibbs energy

\footnotetext{
S. Alkama ( $\square)$

Department of Automatics, University M. MAMMERI,

15000 Tizi-Ouzou, Algeria

e-mail: sadia_alkama@yahoo.fr

Y. Chahir

GREYC, UMR CNRS 607, University of Caen, Campus II,

BP 5186, 14032 Caen Cedex, France

e-mail: Youssef.Chahir@info.unicaen.fr

D. Berkani

Department of Electronics, Polytechnic National School, 16200 El Harrach, Algeria

e-mail: dberkani@hotmail.com
}

\section{Introduction}

Image segmentation means the partitioning of an image into meaningful regions based on homogeneity or heterogeneity criterion, respectively [8]. It represents the interface between image pre-processing and image understanding (object recognition). The significant improvement realized in multispectral radiometer/imager of remote sensing allows multidimensional images with a great of information acquisition.

The segmentation of these images became difficult because of the issues in processing of the great number of multidimensional data. Three approaches namely, marginal, scalar and vector approaches are applicable to these multispectral images.

The marginal approach, in this case, will consist of the segmentation of each spectral component then in the fusion of the images of the labels in only one.

In the scalar approach, we initially make fusion of the various spectral components in only one component, and then we segment this one as a gray-level image.

In the vector approach, the segmentation is directly made on the multispectral image by using vector description of each pixel. Although, the last approach seems to be the first time more complicated to implement and slower than the foregoing ones. However, the vector approach takes into account the interactions of the diverse spectral components of the image that makes the method capable to perform good results. This is the main advantage of the chosen approach to be applied in our research.

In this paper, we are concerned with real images, then natural ones. The nature is not entirely "unforeseeable", two observations very distant one from the other do not in average have an influence between them, while two near observations have more influence between them than they 
are near. This concept is enough similar to the Markov property, witch stipulates that the conditional probability of the value of one site depends on the values of its neighborhoods sites. Thus, the probability that a pixel belongs to a class depends not only on its intensity, but also on those of its neighbors [1,7]. Therefore, we use in this work, a vector approach of segmentation by Markov fields which take into account the spectral and space interactions between the pixels of Meteosat Second Generation (MSG) images. Most of MSG channels are the measurements of infrared radiance which are in practice the brightness temperatures measurements of clouds, land and sea surfaces $[9,18]$. For the purpose for estimate some meteorological parameters (like precipitations, direction and speed of winds, etc.), it is necessary to, initially, extract and characterize the clouds present in these images. This operation will be doing by classifying the various elements contained in those. Image segmentation is recently widely used in remote sensing especially since the availability of very high resolution imagery. Numerous approaches of remote sensing image segmentation based on ant colony [17], mean shift clustering [4, 23], watershed [10, 15], fuzzy C-means clustering [6], memetic [10], Fractal and MRF [13, 21, 22] algorithms have been developed.

For example, Tso and Olsen [22] apply a MRF to segment IKONOS satellite images in order to mapping the Elkhorn natural reserve in Californian coast. For optimization, they use the Maximization of A Posteriori probability (MAP) and for initialization, they utilize a wavelets pre-classification.

Also Keuche et al. [13] use a MRF for segmentation of Landsat 5 images in the purpose to mapping Tenerife of the Canaries islands. A deterministic approach by Iterated Conditional Modes (ICM) algorithm is used to optimize the posterior probability. Markov classification follows a presegmentation by two methods of supervised classification using the ground data. The results, in this case, are improved for a small number of algorithm iteration, but they are degraded when this number becomes significant because more of small regions are deleted or ignored.

Usually, when using the Markovian segmentation, the image processing is modeled by a Gaussian energy, and the label processing is modeled by a Gibbs energy. Gibbs energy which is frequently used is the Pappas energy. In this paper, we propose to test the best known of Gibbs energies. The experimental results were analyzed and evaluated by using the Borsotti function [3].

The remainder paper is organized as follows. In Sect. 2, the MRF unsupervised multi-components segmentation is given in detail. The criterion used to evaluate the different results is described in Sect. 3. Section 4 presents the experimental results. Finally, Sect. 5 culminates with some conclusion.

\section{Multispectral image MRF segmentation model}

The Markov segmentation is obtained by classifying the pixels into different pixel classes by taking into account at the same time their "intensities" and their neighborhoods. The input image is represented by a set $S$ of sites (pixels) $s$ which can be indicated by their sequence number $S=\left\{s_{1}\right.$, $\left.s_{2}, \ldots, s_{M}\right\}$, such as $M=N \times P$ is the cardinal of $S$. For each site $s$ is associated a gray-levels vector $a_{s}$ of a set $\Psi$ of gray-levels of the various spectral channels. The elements of $\Psi$ are $D$ components vectors, such as $D$ is the number of spectral channels. For each site $s$ is also associated a label $\lambda_{s}$. Values taken by $\lambda_{s}$ depend on the number of classes present in this input image.

We suppose that the input multispectral image $a$, with $a=\left\{a_{s}, s \in S\right\}$, is a Markov field $A$. The segmented image, given by a labels image $\lambda$, with $\lambda=\left\{\lambda_{s}, s \in S\right\}$, is also modeled as Markovian field $\Lambda$. The overall segmentation model is composed of the hidden label process $\lambda$ and the observable noisy image process $a$. We can use a Bayesian approach which consists in search of the most probable class by maximize the posterior probability $P(\lambda \backslash A)$. We build $P(a \backslash \lambda)$, then we must fix a prior distribution on $\Lambda$, noted $P(\lambda)$, after, we can calculate $P(\lambda l a)$ by using the Bayes formula.

The posterior probability is then expressed:

$P(\lambda \backslash a)=\frac{P(a \backslash \lambda) P(\lambda)}{P(a)}$

where $P(\lambda \backslash a)$ is the posterior probability; $P(a \backslash \lambda)$ represents the conditional probability of the observation $a$ knowing the class $\lambda ; P(\lambda)$ the probability associated with the field $\Lambda$, called a prior distribution; and $P(a)$ is the constant because it is a realization of the observed process.

The constant factor $1 / P(a)$ will drop because we are only interested in $\lambda$ witch maximize the posterior probability. For maximize $P(\lambda \backslash a)$, we must just maximize $P(a \backslash \lambda) P(\lambda)$.

\subsection{Image processing}

When we have no enough information on the nature of the observation distribution, it usual to use the Gaussian distribution for modeling conditional probability $P(a \backslash \lambda)$ $[5,19,22]$. The multidimensional Gaussian distribution is given by the relation:

$P\left(a_{s} / k\right)=\frac{1}{\left(2 \pi\left|\sigma_{k}\right|\right)^{D / 2}} \exp \left(-\frac{1}{2}\left(a_{s}-\mu_{k}\right)^{T} \sigma_{k}^{-1}\left(a_{s}-\mu_{k}\right)\right)$

where $a_{s}$ is the vector of different channels values of the site $s$ in input image; $\sigma_{k}$ is the covariance matrix between the various components for the class $k ; \mu_{k}$ is mean vector 
for pixel class $k$; and $D$ is the number of spectral channels present in the input image.

To calculate this probability, it is necessary to know the mean vector and covariance matrix of each class.

\subsection{Label processing}

A random field of the labels $\Lambda$ is random Markov field operating in a neighboring system, then:

$P\left(\lambda_{s} \backslash \lambda_{t}, \forall t \in S, t \neq s\right)=P\left(\lambda_{s} \backslash \lambda_{t}, \forall t \in V_{s}\right)$

The Hammersley-Clifford theorem [1, 7] establishes equivalence between Markov field and Gibbs field. Therefore a Markov field which verifies the condition of positiveness is also a Gibbs distribution. Then:

$P\left(\lambda_{s}\right)=\pi\left(\lambda_{s}\right)=\frac{1}{Z} \exp \left(-E\left(\lambda_{s}\right)\right)$

where $E\left(\lambda_{s}\right)$ is called an energy function of the Gibbs field $\Lambda$ defined by:

$E\left(\lambda_{s}\right)=\sum_{c \in C} v_{c}\left(\lambda_{s}\right)$

$Z>0$ is the normalizing constant and is given:

$Z=\sum_{\lambda \in \Omega} \exp (-E(\lambda))$

$v_{\mathrm{c}}$ is the potential function of a clique $c$ and $C$ is the set of cliques corresponding on neighborhood selected.

A clique $\mathrm{c}$ is a sub-set of sites which are mutually neighbored relatively to the neighborhood system defined. The order of one clique corresponds to the number of its sites [5]. In this paper, the neighborhood system used is the 8 -nearest and the order of clique utilized is 2 .

The choice of energy function can be important to get good results with a given classification algorithm. The formula defining energy should allow correct classification without involving significant costs in computation time. There are several models in the literature of these functions. We will, in this work, use five of them and we propose two others. Thereafter, we compare the results obtained.

The first function used is named Pappas [20] function. It is also know the Potts model in statistical physics. It is given by the relation:

$E 1\left(\lambda_{s}\right)=\sum_{c \in C} v_{c}\left(\lambda_{s}\right)=\sum_{i} \sum_{j} \beta_{i, j}$

with $\{i, j\} \subset C$ i.e. $\{i, j\}$ is a clique corresponding to a pairs of neighboring pixels and corresponding in the neighborhood of $s$. The clique potential $\beta_{i, j}$ is given by:

$\beta_{i, j}= \begin{cases}+\beta & \text { if } \lambda_{i} \neq \lambda_{j} \\ -\beta & \text { if } \lambda_{i}=\lambda_{j}\end{cases}$ where $\beta$ is a weighting positive parameter controlling the importance of the prior. As $\beta$ increases, the resulting of regions become more homogeneous. Pappas chooses $\beta=0.5$ [20]. It is this value which will be retained. This potential function imposes a space connectivity constraint for a classification. The assignment of this potential give a high probability for pairs pixels having the same labels and a weak probability for pair pixels having different labels.

To generalize the writing, several functions are combined in a single formula, which is given as follows:

$E 2\left(\lambda_{s}\right)=\sum_{c \in C} V_{c}\left(\lambda_{s}\right)=\beta \sum_{i} \sum_{j}\left|\lambda_{i}-\lambda_{j}\right|^{p}$

where $\beta$ is a regularization smoothing parameter and $p$ is the power of function.

When $p=1$, the function is named the function value of Lamotte and Alt [14], and when $p=2$, it is named a square function of Blanc-Ferraud and Baralaud [2]. We propose in this paper, to study another value, $p=1 / 2$.

Another function used by Lamotte and Alt [14] is:

$E 3\left(\lambda_{s}\right)=\sum_{c \in C} V_{c}\left(\lambda_{s}\right)=\beta \sqrt{\frac{\sum_{i} \sum_{j}\left|\lambda_{i}-\lambda_{j}\right|}{c s t}}$

cst is a normalization constant.

The function proposed by Geman and Gemain [7] is:

$E 4\left(\lambda_{s}\right)=\sum_{c \in C} \mathrm{~V}_{c}\left(\lambda_{s}\right)=\beta \sum_{i} \sum_{j} \frac{\left(\lambda_{i}-\lambda_{j}\right)^{2}}{1+\left(\lambda_{i}-\lambda_{j}\right)^{2}}$

To model rainfall, spherical functions are often used [11]. Since rainfall is closely related to clouds, we propose in this paper one spherical function that has the following form:

$$
\begin{aligned}
E 5\left(\lambda_{s}\right) & =\sum_{c \in C} V_{c}\left(\lambda_{s}\right) \\
& =\beta \sum_{i} \sum_{j}\left|1.5\left(\lambda_{i}-\lambda_{j}\right)-0.5\left(\lambda_{i}-\lambda_{j}\right)^{3}\right|
\end{aligned}
$$

The value 1 is assigned to the regularization smoothing parameter in potential functions $E 2, E 3, E 4$, and $E 5$.

\subsection{Optimization}

Reminded that for maximize $P(\lambda \backslash a)$, it is enough to maximize $P(a \backslash \lambda) P(\lambda)$.

$\hat{\lambda}=\arg \left(\max _{\lambda \in \Omega} P(\lambda \backslash a)\right)=\arg \left(\max _{\lambda \in \Omega} P(a \backslash \lambda) P(\lambda)\right)$

To reduce computation, we note:

$U_{1}(a \backslash \lambda)=-\operatorname{Ln} P(a \backslash \lambda)$

$U_{2}(\lambda)=-\operatorname{Ln} P(\lambda)$ 
The MAP estimation becomes:

$$
\begin{aligned}
\hat{\lambda} & =\arg \left(\min _{\lambda \in \Lambda}\left(U_{1}(a / \lambda)+U_{2}(\lambda)\right)\right) \\
& =\arg \left(\min _{\lambda \in \Lambda}\left(U_{G}(\lambda, a)\right)\right)
\end{aligned}
$$

Thus, it is clear that maximize posterior probability is equivalent to minimize global energy $U_{G}$, with:

$$
\begin{aligned}
U_{G}\left(k, a_{s}\right) \approx & \frac{1}{2}\left(a_{s}-\mu_{k}\right)^{T} \sigma_{k}^{-1}\left(a_{s}-\mu_{k}\right)+\frac{D}{2} \operatorname{Ln}\left(2 \pi\left|\sigma_{k}\right|\right) \\
& +\sum_{c \in C} V_{c}\left(\lambda_{s}\right)
\end{aligned}
$$

Now the segmentation problem is reduce to the minimization of the above function. It can be achieved by stochastic methods or by deterministic methods. Stochastic methods have a great computing time. Deterministic iterative methods do not provide always the global optimum. However they are more used because they have a good trade-off between quality and computing time. Among deterministic algorithms, we chose ICM algorithm [1].

\section{Evaluation criterion}

There are two types of approach to evaluate the results of image segmentation. The first one evaluates the quality of the segmentation result by measuring its similarity with a "ground truth". This "ground truth" is often subjective, especially in the case of the real images. Moreover, it requires having at disposal an expert of the applicative field. The second one is based on unsupervised evaluation criterions, where the quality of segmentation results is estimated from statistics calculated on each detected area. Several of these criterions are developed for gray level images segmentation and few of them are devoted for color images segmentation [24, 25]. The criterions developed for the color imagery can be applied to the multispectral imagery by extending the dimension. One of the first criterion which is proposed for the color imagery is the Liu and Yang function [16]. Borsotti et al. [3] and Zhang [24] showed that this function tends to evaluate very noisy segmentations favorably when the number of regions built is important. Liu and Yang function was improved by operating an adjustment on some parameters [3]. The most important inconvenient of this revised evaluation function is that it is not sensitive enough to small segmentation differences [3, 24, 25].

In this paper, we have chose to use the Borsotti et al. [3] function because it don't require any parameters adjustment, it avoids the inconvenient of the Liu and Yang function and its revised version and it gives an indication on the quality of the results which is in adequacy with our visual perception. It is defined by:

$C r=\frac{\sqrt{N R}}{10^{4} M} \sum_{r=1}^{N R}\left(\frac{E_{r}^{2}}{1+\log \left(\operatorname{cardB}_{r}\right)}+\frac{R\left(B_{r}\right)^{2}}{\operatorname{cardB}_{r}^{2}}\right)$

where $N R$ is the number of regions of the segmented image; $M$ the image size; $E_{r}$ the sum of the distances between the pixels of the area $B_{r}$ in input image and the multi-component value attributed to the area $B_{r}$ in the segmented image; and $R\left(B_{r}\right)$ is the number of regions having exactly an area equal to that of $B_{r}$.

The first term of the sum is high only for non-homogeneous regions (typically, large ones), while the second is high only for regions whose area $B_{r}$ is equal to the area of many other regions in the segmented image (typically, small ones). This function penalizes segmentations that form too many regions and having non-homogeneous regions. More this function present a low value, more the segmentation is of better quality.

\section{Experimental results}

Images used in this paper provided from meteorological satellite "Meteosat Second Generation 2" (MSG 2). The imaging tasks are performed by the radiometer called Spinning Enhanced Visible and Infrared Imager (SEVIRI) which is equipped with 12 spectral channels. It measures then the energy transported by the electromagnetic waves at different spectral frequencies.

Only the five infra-red channels IR87 (8.3-9.1 $\mu \mathrm{m})$, IR97 (9.3-9.94 $\mu \mathrm{m}), \quad$ IR108 $(9.8-11.8 \mu \mathrm{m}), \quad$ IR120 $(11-13 \mu \mathrm{m})$ and IR134 $(12.4-14.4 \mu \mathrm{m})$ were retained in this work because they are exploitable in daytime and in night. Contrary, the visible channels VIS06 $(0.56-0.7 \mu \mathrm{m})$, VIS08 $(0.74-0.88 \mu \mathrm{m})$, high resolution visible channel HRV $(0.4-1.1 \mu \mathrm{m})$ and near infra-red channels NIR16 $(1.5-1.78 \mu \mathrm{m})$, NIR38 $(3.48-4.36 \mu \mathrm{m})$ are exploitable only in daytime because they depend completely on the sun light. In additional, the covered areas are too wide then it is necessary to perform a correction according the sun position in order to have a homogeneous lighting on the entire scene. The water vapor channels VW62 $(5.35-7.17 \mu \mathrm{m})$ and VW73 $(6.85-7.85 \mu \mathrm{m})$ were not retained also because they do not characterize the convective clouds which are the most important meteorological atmospherics phenomena observed by satellites. With these five infra-red channels, we can hope for a good detection of the convective clouds [11]. Each channel of these MSG2 images study constitutes a spectral component which can be regarded as a gray-level image, coded on 8 bits and have $600 \times 500$ pixels $^{2}$ for dimension. These images describe the area which is 
between $15^{\circ}$ North and $40^{\circ}$ North for latitudes and between $14^{\circ}$ West and $16^{\circ}$ East for longitudes.

The Markov segmentation described by ICM algorithm requires initial classification of the pixels of the input images. This initial classification of the input image is, in the major cases, done by the "Expectation-Maximization" (EM) algorithm [5, 12]. This algorithm is also sensible to initial conditions. As the goal of our work is to compare the results when we use different form of Gibbs energy, the segmentation results must be independent of the initialization step. Hence, we propose a simple and faster initial classification for the ICM algorithm. The initial classification is computed from the component named IRmoy which corresponds to the arithmetic mean of these various

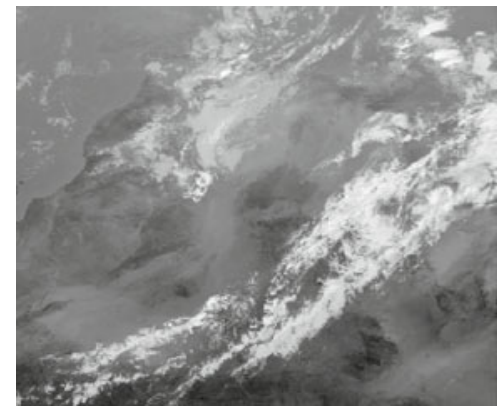

(a1)

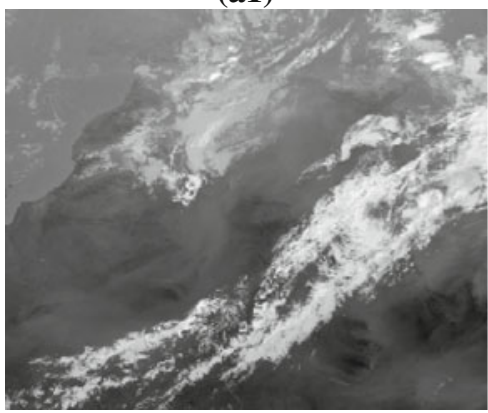

(d1)

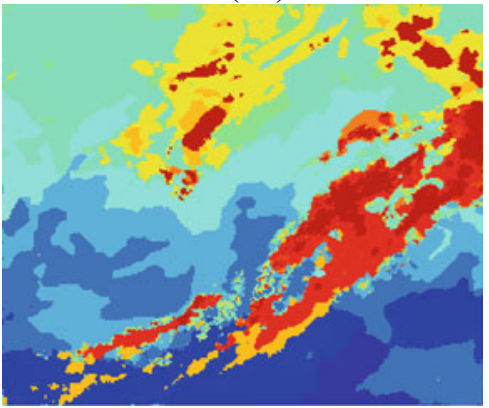

(b2)

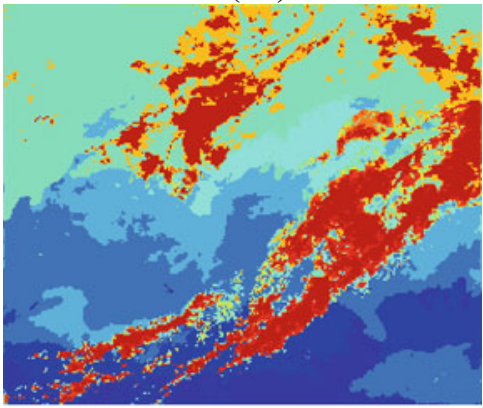

(e2)

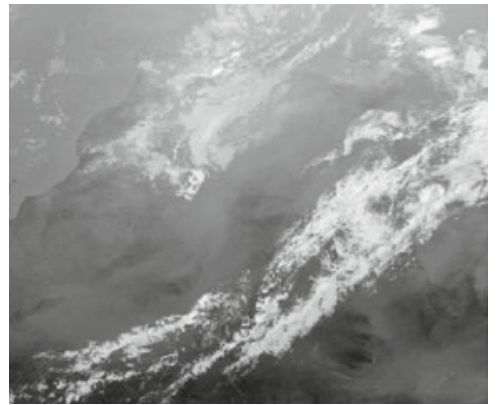

(b1)

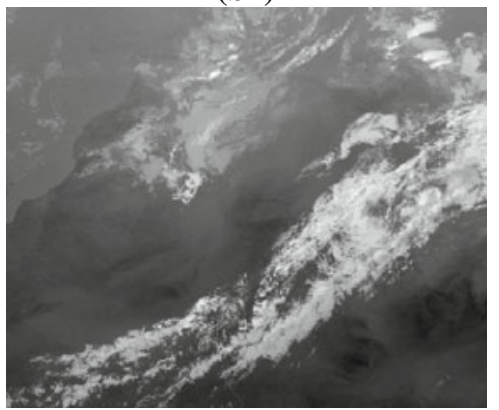

(e1)

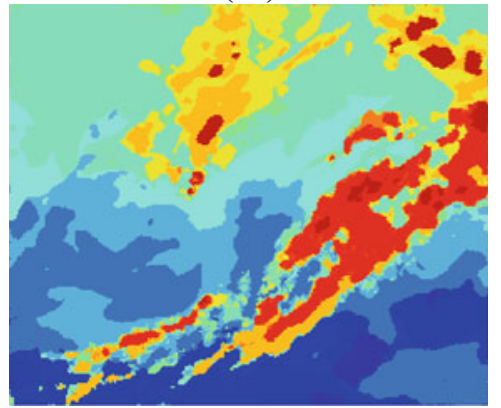

(c2)

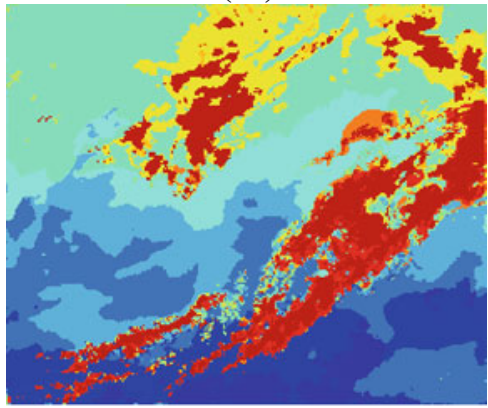

(f2)

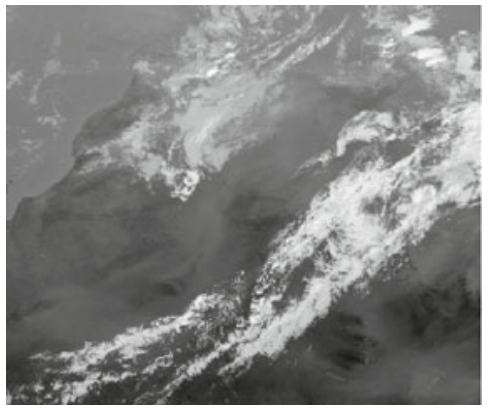

(c1)

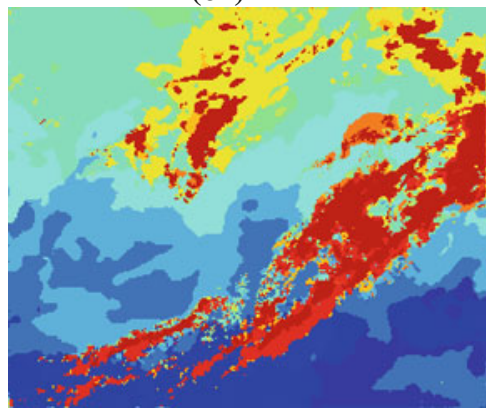

(a2)

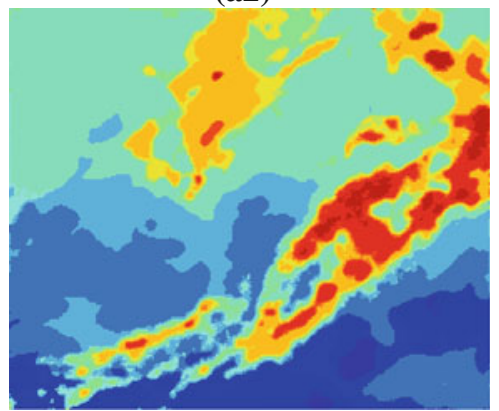

(d2)

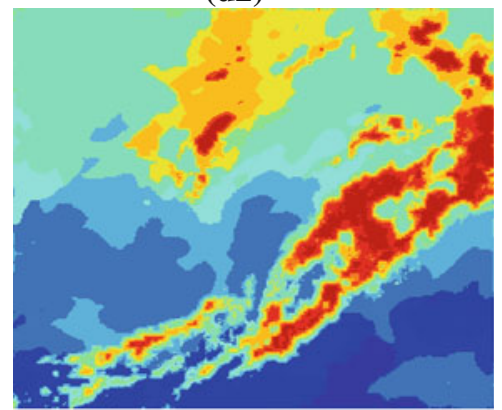

(g2)
Fig. 1 Segmentation of the multispectral image recorded in January 16, 2007 at noon $(K=15$, Itc $=10)$. Image components: a1 IR087, b1 IR097, c1 IR108, d1 IR120, e1 IR134; Segmentation results by using: a2 E1 energy, b2 E2 ( $p=1 / 2)$ energy, $\mathbf{c 2} E 2(p=1)$ energy, d2 $E 2(p=2)$ energy, e2 $E 3$ energy, f2 $E 4$ energy and g2 $E 5$ energy 
components of a multispectral image. This method consists to divide the histogram dynamics of the IRmoy component in equal intervals. The pixels witch have a gray-levels in the same interval are assigned to the same class. The number of intervals is then equal to the number of desired classes $K$. After this initial segmentation, the Markov procedure is applied by taking into account all the five spectral components of the input image.

Figure 1 illustrates an example of the segmentation results of the multispectral image MSG2. The two first lines in this figure give the various IR channels of this image. Below, we have the results of multi-component classification with different form of Gibbs energy. All the results of this figure are provided for a number of classes $K$ equal to 15 and number of iteration count Itc equal to 10 . Itc is the criterion used to stop Iterated Conditional Modes (ICM) algorithm. Effectively, in this algorithm, the stop criterion can be, either a number of iterations Itc fixed by the user, or a threshold not exceeded on number of pixels having changed class to the last iteration. Generally, this threshold is $10 \%$ of pixels number in the image [7]. The labels images are displayed in color in order to well separate the classes. The representation of the various channels is giving in gray-levels because the dynamics of these is of 256 levels. We give for each segmentation result the value of the Borsotti function $\mathrm{Cr}$, the number of joined area $\mathrm{NR}$ detected and the percentage of pixels changed classes in the last iteration $\mathrm{Pr}$ in Table 1. All these results are given for a number of iteration of the algorithm equal to 10 .

Observing the results displayed on Table 1 , we notice that broadly the values obtaining for Borsotti function are appreciable because they are comparable with those provided by Borsotti et al. [3]. Indeed, $\mathrm{Cr}$ stay lower than 10000 . It is necessary to note that more the number of used channels increases, more the value of Borsotti function will be important, for the same segmentation quality,

Table 1 Results of the tests for three images

\begin{tabular}{|c|c|c|c|c|c|c|c|c|c|}
\hline \multirow[t]{2}{*}{ Gibbs energies } & \multicolumn{3}{|c|}{ Image $1^{\mathrm{a}}, K=15$} & \multicolumn{3}{|c|}{ Image $2^{\text {a }}, K=15$} & \multicolumn{3}{|c|}{ Image $3^{\mathrm{a}}, K=15$} \\
\hline & $P r$ & $\mathrm{Cr}$ & $N R$ & $\operatorname{Pr}$ & $\mathrm{Cr}$ & $N R$ & $\operatorname{Pr}$ & $\mathrm{Cr}$ & $N R$ \\
\hline E1 & 2.90 & 758 & 933 & 4.38 & 339 & 1160 & 2.62 & 860 & 1215 \\
\hline$E 2, p=1 / 2$ & 3.48 & 959 & 857 & 4.54 & 350 & 1214 & 2.45 & 713 & 1076 \\
\hline$E 2, p=1$ & 3.81 & 1076 & 842 & 4.42 & 477 & 1267 & 2.70 & 664 & 901 \\
\hline$E 2, p=2$ & 4.13 & 1396 & 1331 & 4.73 & 983 & 1306 & 3.94 & 725 & 740 \\
\hline E3 & 2.49 & 2054 & 1716 & 5.13 & 1339 & 3336 & 2.84 & 1252 & 2134 \\
\hline E4 & 2.57 & 946 & 1078 & 5.48 & 580 & 2059 & 2.66 & 920 & 1401 \\
\hline E5 & 3.78 & 1297 & 791 & 6.05 & 336 & 1261 & 4.13 & 758 & 674 \\
\hline
\end{tabular}

${ }^{a}$ Meteosat image by Jan. 16, 2007 at noon (image 1), Dec. 15, 2006 at noon (image 2) and Nov. 15, 2006 at noon (image 3)

$K$ Number of classes; $\operatorname{Pr}$ percentage of pixels changed classes in the last (10th) iteration of ICM algorithm; $C r$ value of Borsotti function; $N R$ number of joined area in segmented image because the distance $E_{r}$ (see Eq. 17) will become more significant. Indeed, this distance is computed by adding the Euclidean distances between the pixels values in input images and the multi-component value attributed in the segmented image. Then more we have component, more we have difference terms to add in the square, then more this distance will be great. Considering that the value of Borsotti function for a good classification of color image, which is on three components does not exceed 10,000 and considering that the treated images in this paper are on five components and the seeing the various results of $\mathrm{Cr}$ given in Table 1, we can conclude that the MSG images segmentation results are relatively very good.

The labels images obtained by using a multi-component classification (Fig. 1) are different when we use different Gibbs energy, but the clouds are well detected and the clouds of different densities are well separate in the whole of the results (they are the classes which are represented in orange and red colors).

In this work, seven Gibbs energy function are tested, among them, two are proposed, E2 (Eq. 9) with $p=1 / 2$, and $E 5$ (Eq. 12). It is not possible to choose the best energy function because that which provide the best result is different from an image to another. Energy function E3 (Eq. 10) gives the worse results in all tests we did, that can be clearly noted in Table 1 . The proposed energy E2 $(p=1 / 2)$ is always among the three bests results.

The maximum iteration count was fixed at 10 . This number is largely sufficient because the maximum value we found for $\operatorname{Pr}$ after 10 iteration is $6.05 \%$ (it is obtained for image by December 15, 2006 at noon), which is largely sufficient since it is lower than $10 \%$.

To have an outline of the $\mathrm{Pr}$ evolution according to the iteration count, we display in Fig. 2, the arithmetic mean of

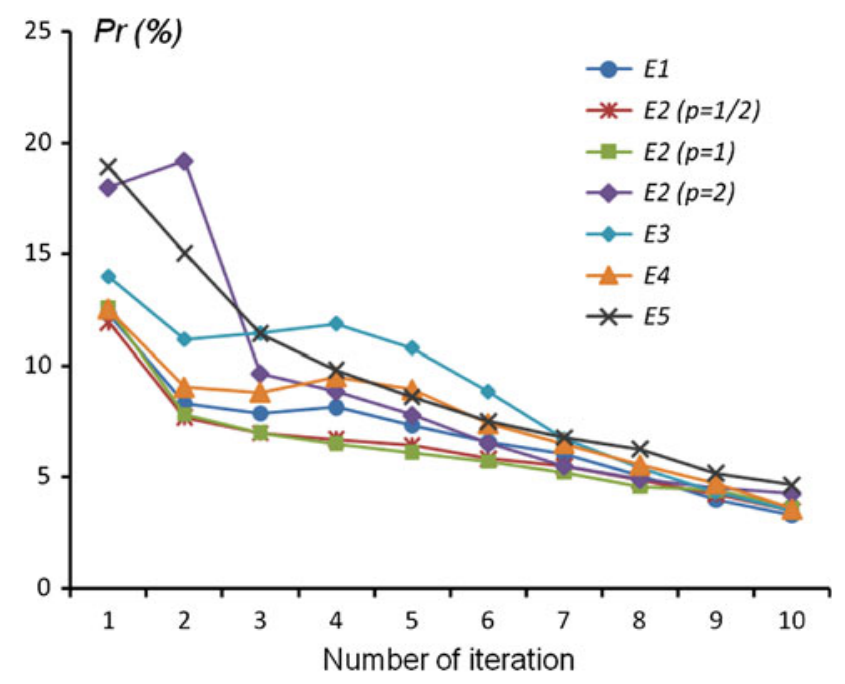

Fig. 2 Variation of the rate of pixels having changed class in $i$ th iteration of ICM algorithm 
this rate, by energy, when the segmentation is carried out on several images.

The convergence is fast since in all the cases, after the third iteration, we obtain $\operatorname{Pr}$ lower than $10 \%$. From the 9th iteration, we obtain in the majority of the cases $\operatorname{Pr}$ lower than 5\%.

Curves are overall decreasing; it may be that they have local maximum but after they decrease continuously.

On all tested examples, algorithm never diverges, even for a very significant number of iterations. It can oscillate slightly and locally on some iterations but it continues overall to converge by decreasing Pr. To have an idea of evolutions and changes undergoes with iterations we visualize on Fig. 3 the multispectral segmentation results of January 16, 2007 image unregistered at noon by using $E 1$ (Eqs. 7 and 8) Gibbs energy. On this last figure, we see that, more the iteration count increases, more the small and isolated areas are eliminated. This result is expected because for classifying one pixel in Markovian segmentation we take

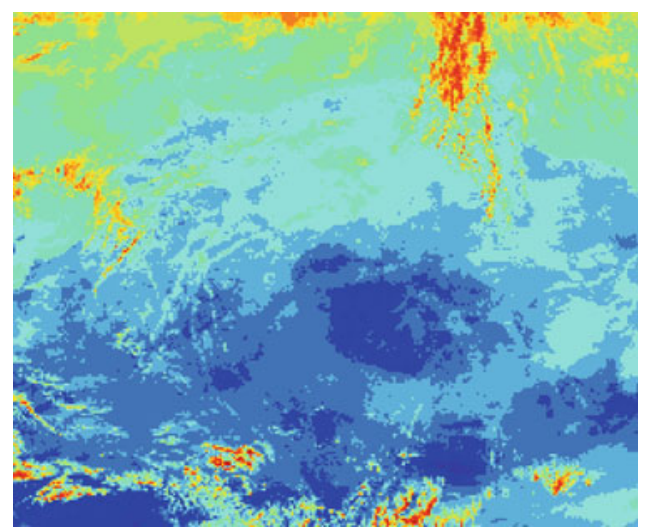

(a)

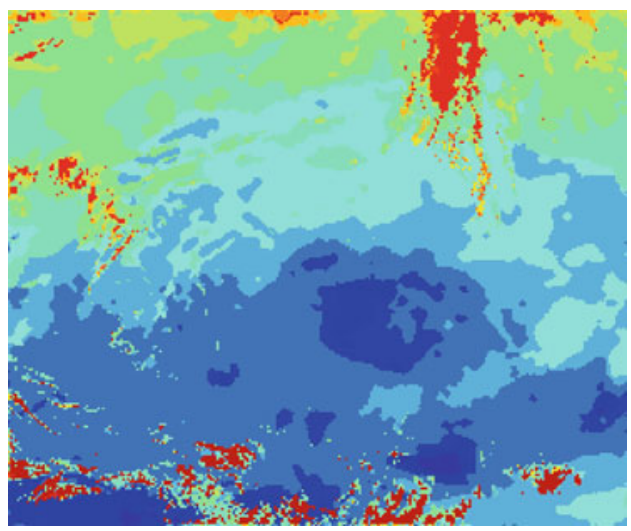

(c)

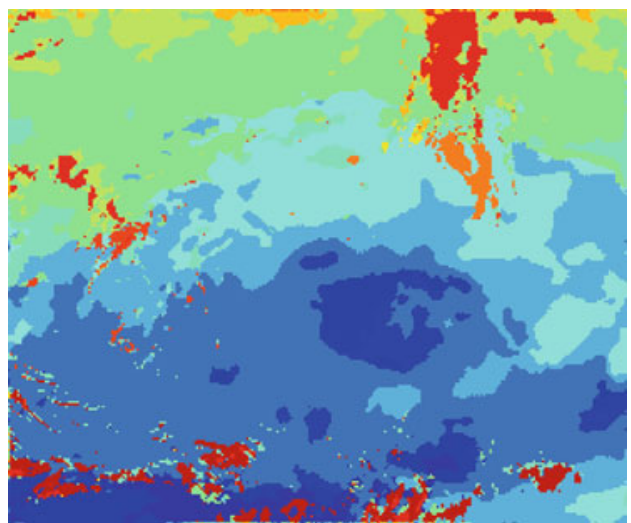

(e)

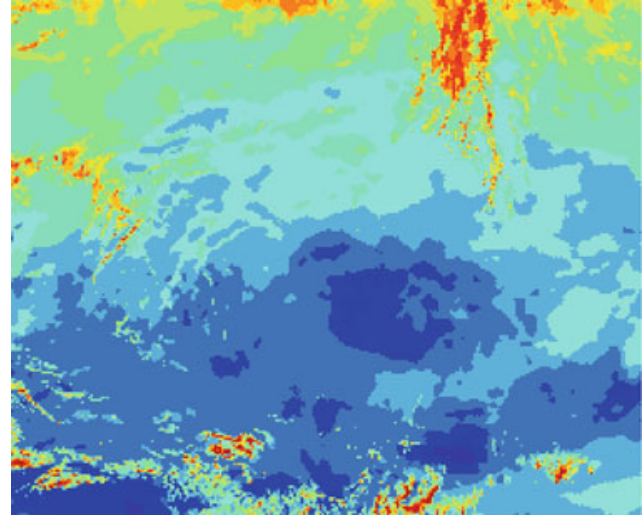

(b)

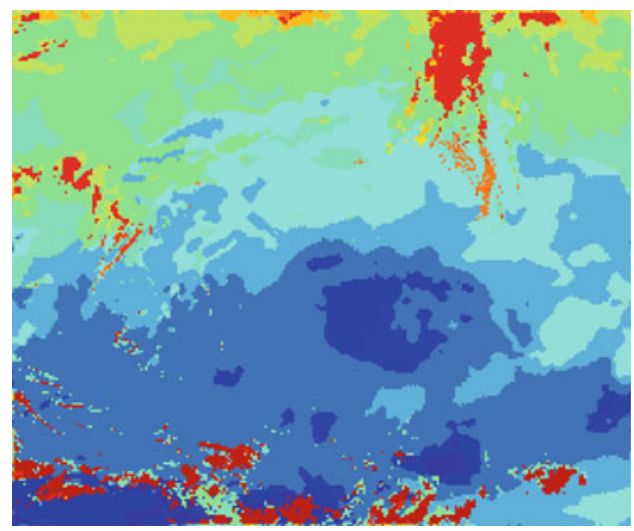

(d)

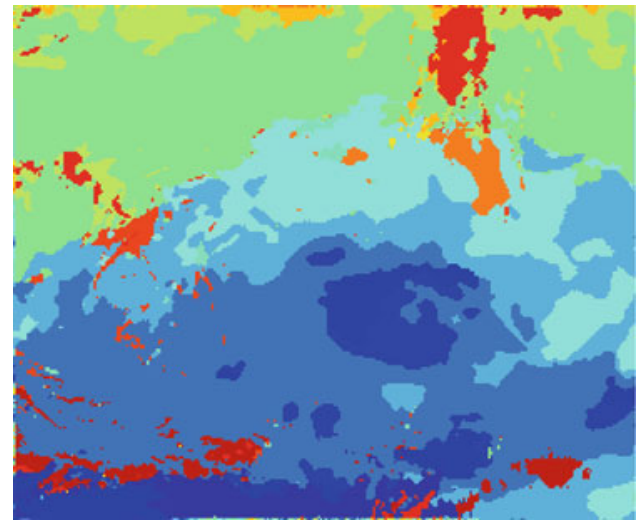

(f)

Fig. 3 Evolution of results with the iteration number in segmenting image recorded in December 16, 2007, for $K=15$ and $E 1$ energy. a Initial classification, b 2, c 5, d 7, e 10 and $\mathbf{f} 20$ iterations 
into count the information of its neighborhoods. This characteristic is generally appreciable when we treat real images. Indeed atmospherics phenomena are rarely isolated and restricted. We tested the case of 20 iterations for multispectral image segmentation of the day January 16, 2007. We find at the 20th iteration $\mathrm{Pr}=0.54 \%$ and $\mathrm{Cr}=749$ against $\mathrm{Pr}=2.9 \%$ and $\mathrm{Cr}=758$ for the 10 th iteration. After the 10th iteration we do not note clear modifications of the segmentation results. That is clearly visible in Fig. 3 where the results of the segmentation to the 10th and the 20th iteration are displayed.

\section{Conclusion}

In this article, we presented a method of multi-component segmentation of MSG images based on Markov formalism. In this approach where the space and spectral interactions between pixels are taken into account, the pixels classification is making by using the Maximum A Posteriori probability (MAP). For obtaining the most probable classification by using the MAP, it is necessary to model the observations field (image process) and the classes field (label process).

The label process is modeling by using Gibbs energy. Seven forms of Gibbs energy are compared in the multispectral segmentation framework. Two of them are newly proposed in this paper. The results show that the choice of Gibbs energy function have an influence on the segmentation quality, although this influence is not very significant. The proposed functions give good results.

For the image process, only the multidimensional Gaussian distribution is used as model in this paper. Others forms of distribution which are not necessarily Gaussian must be investigated. This constitutes our further work.

The results are evaluated with Borsotti function which is usually known to be reliable. The different tests show that the results are satisfactory. Indeed, the algorithm converges in a small number of iteration, the values of Borsotti function are acceptable and the clouds are suitably detected and well separated. Therefore to ensure the algorithm convergence towards the global optimum we can use simulated annealing algorithm.

\section{References}

1. Besag, J. (1974). Spatial interaction and the statistical analysis of lattice systems. Journal of the Royal Statistical Society: Series B, 36, 192-236.

2. Blanc-Ferraud, L., \& Baralaud, M. (1990). Restauration d'images bruitées par analyse multi résolution et champs de Markov. Traitement du Signal, 10(2), 171-184.
3. Borsotti, M., Campadelli, P., \& Schettini, R. (1998). Quantitative evaluation of color image segmentation results. Pattern Recognition Letters, 19, 741-747.

4. Dai, Q., Liu, G., Wang, C., \& Wang, L. (2008). A remote sensing image segmentation method based on spectral and structure information fusion. The international archives of the photogrammetry. In Remote sensing and spatial information sciences (Vol. 38), Beijing.

5. Deng, H., \& Clausi, D. A. (2004). Unsupervised image segmentation using a simple MRF model with a new implementation scheme. Pattern Recognition, 37, 2323-2335.

6. Fan, J., Han, M., \& Wang, J. (2009). Single point iterative weighted fuzzy C-means clustering algorithm for remote sensing image segmentation. Pattern Recognition, 42(11), 2527-2540.

7. Geman, S., \& Gemain, D. (1984) Stochastic relaxation, Gibbs distribution, and the Bayesian restoration of images. IEEE Transactions on Pattern Analysis and Machine Intelligence, PAMI-6(6), 721-741.

8. Haralick, R. M., \& Shapiro, L. G. (1992). Computer and robot vision (Vol. 1). Reading, MA: Addison Wesley.

9. Hasler, A. F., Stong, J., Woodward, R. H., \& Pierce, H. (1991). Automatic analysis of stereoscopic satellite image pairs for determination of cloud-top height and structure. Journal of Applied Meteorology, 30, 257-281.

10. Jiao, L., Gong, M., Wang, S., Hou, B., Zheng, Z., \& Wu, Q. (2010). Natural and remote sensing image segmentation using memetic computing. Computational Intelligence Magazine IEEE, 5(2), 78-91.

11. Jobard, I., \& Desbois, M. (1992). Remote sensing of rainfall over tropical Africa using Meteosat infrared imagery: sensitivity to time and space averaging. International Journal of Remote Sensing, 13(14), 2683-2700.

12. Kato, Z., \& Pong, T. C. (2006). A Markov field image segmentation model for color textured images. Image and Vision Computing, 24, 1103-1114.

13. Keuche, J., Naumann, S., Heiler, M., \& Siegmund, A. (2003). Automatic land cover analysis for Tenerife by supervised classification using remotely sensed data. Remote Sensing of Environment, 86(4), 530-541.

14. Lamotte, R., \& Alt, R. (1994). Comparison of simulated annealing algorithm for image restoration. Mathematics and Computers in Simulation, 37, 1-15.

15. Li, D., Zhang, G., Wu, Z., \& Yi, L. (2010). An edge embedded marker-based watershed algorithm for high resolution remote sensing image segmentation. IEEE Transactions on Image Processing, 19(10), 2781-2787.

16. Liu, J., \& Yang, Y.-H. (1994). Multiresolution color image segmentation. IEEE Transactions on Pattern Analysis and Machine Intelligence, 16(7), 689-700.

17. Mei, X., Wang, Q., Wang, Q., \& Lin, W. (2009). Research on remote sensing image segmentation based on ant colony algorithm-take the land cover classification of middle Qinling Mountains for example. In International symposium on multispectral image processing and pattern recognition, No. 6, Yichang Shi, China.

18. Nieman, S. J., Schmetz, J., \& Menzel, W. P. (1993). A comparison of several techniques to assign heights to cloud tracers. Journal of Applied Meteorology, 32, 1559-1568.

19. Panjwani, D. K., \& Healey, G. (1995). Markov random field models for unsupervised segmentation of textured color images. IEEE Transactions on Pattern Analysis and Machine Intelligence, 7(10), 939-954.

20. Pappas, T. (1992). An adaptive clustering for image segmentation. IEEE Transactions on Signal Processing, 40(4), 901-914.

21. Poggi, G., Scarpa, G., \& Zerubia, J. B. (2005). Supervised segmentation of remote sensing images based on a tree-structured 
MRF model. IEEE Transactions on Geosciences and Remote Sensing, 43(8), 1901-1911.

22. Tso, B., \& Olsen, R. C. (2005). A contextual classification scheme based on MRF model with improved parameter estimation and multi-scale fuzzy line process. Remote Sensing of Environment, 97(1), 127-136.

23. Zhanfeng, S., Jiancheng, L., Xiaodong, H., \& Weigang, S. (2010). A mean shift multi-scale segmentation for remote sensing images. Geomatics and Information Science of Wuhan University, 35(3), 313-316.

24. Zhang, Y. J. (1996). A survey of evaluation methods of image segmentation. Pattern Recognition, 29(8), 1335-1346.

25. Zhang, H., Fritts, J. E., \& Goldman, S. A. (2008). Image segmentation evaluation: A survey of unsupervised methods. Computer Vision and Image Understanding, 110(2), 260-280.

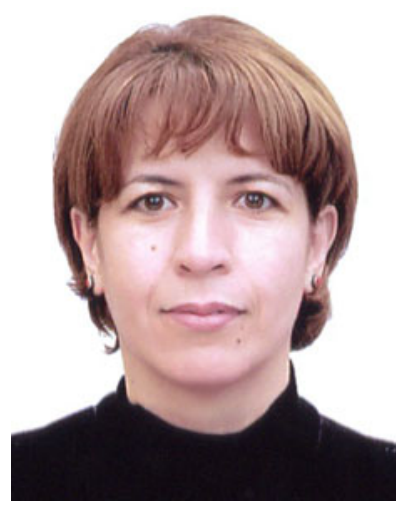

Sadia Alkama has received the Engineering degree in Electronics and the Master of Philosophy degree in Industrial Automatic Control from the University Mouloud Mammeri of Tizi Ouzou, Algeria, in 1993 and 1998 respectively. She is a permanent researcher-teacher in the Automatic Control Department of the Electrical Engineering and Computer Science Faculty of the University Mouloud Mammeri of Tizi Ouzou, Algeria. Her research activity is mainly focused on multi-component image processing and analysis.

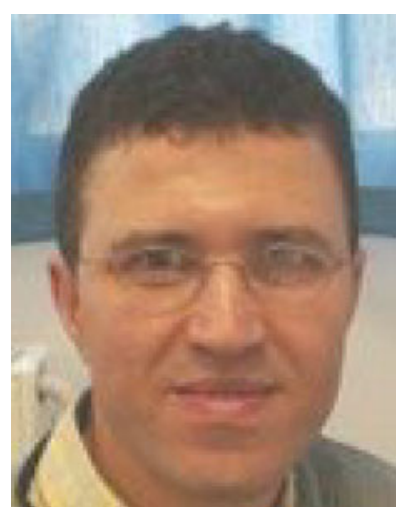

Youssef Chahir received a Ph.D. in Computer Science from Central School of Lyon, France in 2000. Since 2000, he is an assistant professor at the University of Caen in the Computer Science Department. His work is primarily focused on color image segmentation, video analysis and classification methods for pattern recognition.

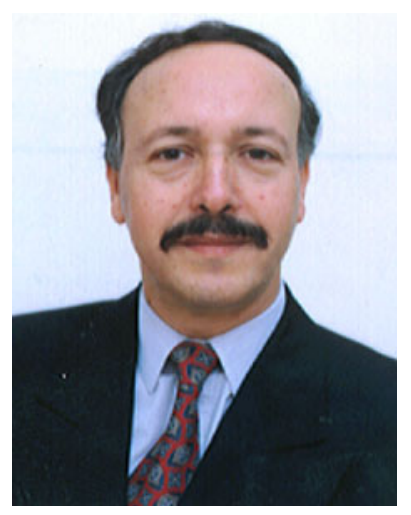

Daoud Berkani received the engineer diploma and Master degree with Red Award from the Polytechnic Institute of Kiev in 1977, then the Magister and Sc.D. degrees from the National Polytechnic School (NPS), Algiers. In 1979, he became Lecturer, Associated Professor then full Professor teaching signal processing and information theory in the Department of Electronics of the NPS. During this period, his research activities involved the applications of signal processing and source coding theory. In 1992, he joined the Department of Electrical Engineering of University of Sherbrook, Canada, where he taught signal processing. He was a member of the speech coding team of the University of Sherbrook. He has been conducting research in the area of speech coding and speech processing in adverse conditions. In 1995, he backs to the Department of Electrical and Computer Engineering of NPS. His current research interests include signal and communications, information theory concepts, clustering and adaptive algorithms, applied to speech and image processing. He is author of more than 150 papers. 